\title{
Cloaking using complementary media in the quasistatic regime
}

\author{
Hoai-Minh Nguyen ${ }^{1}$ \\ EPFL SB MATHAA CAMA, Station 8, CH-1015 Lausanne, Switzerland
}

Received 25 July 2014; received in revised form 5 May 2015; accepted 10 June 2015

Available online 22 July 2015

\begin{abstract}
Cloaking using complementary media was suggested by Lai et al. in [8]. The study of this problem faces two difficulties. Firstly, this problem is unstable since the equations describing the phenomenon have sign changing coefficients, hence the ellipticity is lost. Secondly, the localized resonance, i.e., the field explodes in some regions and remains bounded in some others as the loss goes to 0 , might appear. In this paper, we give a proof of cloaking using complementary media for a class of schemes inspired from [8] in the quasistatic regime. To handle the localized resonance, we introduce the technique of removing localized singularity and apply a three spheres inequality. The proof also uses the reflecting technique in [11]. To our knowledge, this work presents the first proof on cloaking using complementary media.
\end{abstract}

( 2015 L'Association Publications de l'Institut Henri Poincaré. Published by Elsevier B.V. All rights reserved.

MSC: 35B34; 35B35; 35B40; 35J05; 78A25; 78M35

Keywords: Negative index materials; Cloaking; Sign changing coefficients; Localized resonance; Complementary media

\section{Introduction}

Negative index materials (NIMs) were first investigated theoretically by Veselago in [18] and were innovated by Nicorovici et al. in [15] and Pendry in [16]. The existence of such materials was confirmed by Shelby, Smith, and Schultz in [17]. The study of NIMs has attracted a lot the attention of the scientific community thanks to their many possible applications. One of the appealing ones is cloaking. There are at least three ways to do cloaking using NIMs. The first one is based on the concept of anomalous localized resonance discovered by Milton and Nicorovici in [10]. The second one is based on plasmonic structures introduced by Alu and Engheta in [2]. The last one makes use of the concept of complementary media and was suggested by Lai et al. in [8]. In this paper, we concentrate on the last method.

E-mail address: hoai-minh.nguyen@epfl.ch.

1 The research is partially supported by NSF grant DMS-1201370 and by the Alfred P. Sloan Foundation. 
The study of cloaking using complementary media faces two difficulties. Firstly, this problem is unstable since the equations describing the phenomenon have sign changing coefficients, hence the ellipticity is lost. Secondly, the localized resonance, i.e., the field explodes in some regions and remains bounded in some others as the loss goes to 0 , might appear, see [8, Figure 2].

In this paper, we give a proof of cloaking using complementary media for a class of schemes inspired by the work of Lai et al. in [8] in the quasistatic regime. To handle the localized resonance, we introduce the technique of removing localized singularity and apply a three spheres inequality. The proof also uses the reflecting technique in [11]. To our knowledge, this work presents the first proof on cloaking using complementary media.

Let us describe how to cloak the region $B_{2 r_{2}} \backslash B_{r_{2}}$ for some $r_{2}>0$ in which the medium is characterized by a matrix $a$ using complementary media. Here and in what follows given $r>0, B_{r}$ denotes the ball in $\mathbb{R}^{d}(d=2$ or 3$)$ centered at the origin of radius $r$. The assumption on the cloaked region by all means imposes no restriction since any bounded set is a subset of such a region provided that the radius and the origin are appropriately chosen. The idea suggested by Lai et al. in [8] (for two dimensions) is to construct a complementary medium of $a$ in $B_{r_{2}} \backslash B_{r_{1}}$ for some $0<r_{1}<r_{2}$. Inspired by their idea, we construct a cloak in two and three dimensions as follows. Our cloak consists of two parts. The first one, in $B_{r_{2}} \backslash B_{r_{1}}$, makes use of complementary media to cancel the effect of the cloaked region. The second one, in $B_{r_{1}}$, is to fill the space which "disappears" from the cancellation by the homogeneous media. For the first part, we slightly change the strategy in [8]. Instead of $B_{2 r_{2}} \backslash B_{r_{2}}$, we consider $B_{r_{3}} \backslash B_{r_{2}}$ for some $r_{3}>0$ as the cloaked region in which the medium is given by the matrix

$$
b= \begin{cases}a & \text { in } B_{2 r_{2}} \backslash B_{r_{2}}, \\ I & \text { in } B_{r_{3}} \backslash B_{2 r_{2}} .\end{cases}
$$

The complementary medium in $B_{r_{2}} \backslash B_{r_{1}}$ is given by

$$
-\left(F^{-1}\right)_{*} b
$$

where $F: B_{r_{2}} \backslash \bar{B}_{r_{1}} \rightarrow B_{r_{3}} \backslash \bar{B}_{r_{2}}$ is the Kelvin transform with respect to $\partial B_{r_{2}}$, i.e.,

$$
F(x)=\frac{r_{2}^{2}}{|x|^{2}} x
$$

Here and in what follows we use the standard notation

$$
T_{*} b(y)=\frac{D T(x) b(x) D T^{T}(x)}{J(x)} \text { where } x=T^{-1}(y) \text { and } J(x)=|\operatorname{det} D T(x)|,
$$

for a diffeomorphism $T$. It follows that

$$
r_{1}=r_{2}^{2} / r_{3} \text {. }
$$

Concerning the second part, the medium in $B_{r_{1}}$ is given by

$$
\left(r_{3}^{2} / r_{2}^{2}\right)^{d-2} I
$$

The reason for this choice is condition (1.11) (mentioned later in the introduction). Note that in the two dimensional case, the medium in $B_{r_{1}}$ is $I$, as used in [8], while it is not $I$ in the three dimensional case. The cloaking scheme discussed here can be extended for a large class of reflections considered in [11]. The cloaking setting is illustrated in Fig. 1.

To study the problem correctly, one should allow some loss in the medium and study the limit as the loss goes to 0 . With the loss, the medium is characterized by $s_{\delta} A$ where

$$
A=\left\{\begin{array}{cl}
b & \text { in } B_{r_{3}} \backslash B_{r_{2}} \\
F_{*}^{-1} b & \text { in } B_{r_{2}} \backslash B_{r_{1}} \\
\left(r_{3}^{2} / r_{2}^{2}\right)^{d-2} I & \text { in } B_{r_{1}} \\
I & \text { otherwise }
\end{array}\right.
$$




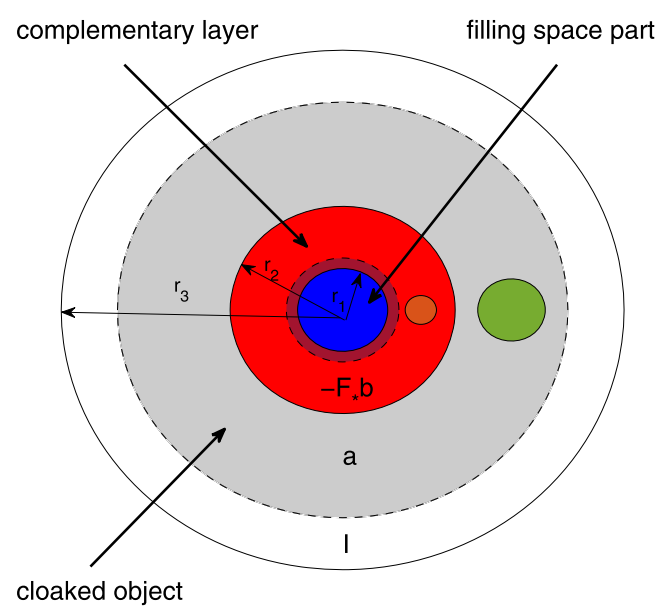

Fig. 1. The cloaked region in $B_{r_{3}} \backslash B_{r_{2}}$ consists of two parts: $a$ in $B_{2 r_{2}} \backslash B_{r_{2}}$ (grey and green regions), which is the original object, and $I$ in $B_{r_{3}} \backslash B_{2 r_{2}}$. The cloaking device contains two parts. The first part $-F_{*} b$ (the red, orange, and pink regions) in $B_{r_{2}} \backslash B_{r_{1}}$ is the complementary medium of $b$ : the red part is complementary to the grey part, the orange part is the complementary to the green part, and the pink part is the complementary to $I$-layer. The second part $\left(r_{3}^{2} / r_{2}^{2}\right)^{d-2} I$ (the blue region) is to fill the space which disappears by the cancellation. (For interpretation of the references to color in this figure, the reader is referred to the web version of this article.)

and

$$
s_{\delta}=\left\{\begin{array}{cl}
-1+i \delta & \text { in } B_{r_{2}} \backslash B_{r_{1}}, \\
1 & \text { otherwise. }
\end{array}\right.
$$

Physically, the imaginary part of $s_{\delta} A$ is the loss of the medium (more precisely the loss of the medium of negative index in $\left.B_{r_{2}} \backslash B_{r_{1}}\right)$. Here and in what follows, we assume that,

$$
\frac{1}{\Lambda}|\xi|^{2} \leq\langle a(x) \xi, \xi\rangle \leq \Lambda|\xi|^{2} \quad \forall \xi \in \mathbb{R}^{d} \text {, for a.e. } x \in B_{2 r_{2}} \backslash B_{r_{2}},
$$

for some $\Lambda \geq 1$. Here and in what follows $\langle\cdot, \cdot\rangle$ denotes the Euclidean scalar product in $\mathbb{R}^{d}$. We require in addition that

\section{$b$ is Lipschitz.}

This paper deals with the bounded setting equipped the zero Dirichlet boundary condition in the quasistatic regime. Let $\Omega$ be a smooth open subset of $\mathbb{R}^{d}(d=2,3)$ such that $B_{r_{3}} \subset \subset \Omega$. Given $f \in L^{2}(\Omega)$, let $u_{\delta}, u \in H_{0}^{1}(\Omega)$ be respectively the unique solution to

$$
\operatorname{div}\left(s_{\delta} A \nabla u_{\delta}\right)=f \text { in } \Omega,
$$

and

$$
\Delta u=f \text { in } \Omega .
$$

Here is the main result of this paper.

Theorem 1. Let $d=2,3, f \in L^{2}(\Omega)$ with supp $f \subset \Omega \backslash B_{r_{3}}$ and let $u, u_{\delta} \in H_{0}^{1}(\Omega)$ be the unique solution to (1.7) and (1.8) respectively. There exists $\ell>0$, depending only on $r_{2}, \Lambda$, and the Lipschitz constant of $b$ such that if $r_{3}>\ell r_{2}$ then

$$
u_{\delta} \rightarrow u \text { weakly in } H^{1}\left(\Omega \backslash B_{r_{3}}\right) \text { as } \delta \rightarrow 0 .
$$

For an observer outside $B_{r_{3}}$, the medium in $B_{r_{3}}$ looks like the homogeneous one by (1.9) (see also (1.8)): one has cloaking. 
One can verify that medium $s_{0} A$ is of reflecting complementary property, a concept introduced in [11, Definition 1], by considering diffeomorphism $G: \mathbb{R}^{d} \backslash \bar{B}_{r_{3}} \rightarrow B_{r_{3}} \backslash\{0\}$ which is the Kelvin transform with respect to $\partial B_{r_{3}}$, i.e.,

$$
G(x)=r_{3}^{2} x /|x|^{2} .
$$

It is important to note that

$$
G_{*} F_{*} A=I \text { in } B_{r_{3}}
$$

since $G \circ F(x)=\left(r_{3}^{2} / r_{2}^{2}\right) x$. This is the reason behind the choice of $A$ in (1.3).

The proof of Theorem 1 is based on two crucial ingredients. The first one is a three spheres inequality. The second one is the technique of removing localized singularity introduced in this paper. ${ }^{2}$ The proof of Theorem 1 is inspired by the approach in [11] where the reflecting technique is used. The removing singularity technique is used in $[12,13]$ to study superlensing and cloaking via anomalous localized resonance.

NIMs have been studied extensively recently see [3-7,9-11,14] and references therein. In [3] and [7], Ammari et al. and Kohn et al. studied the blow up of the power $\left(\sim \delta\left\|u_{\delta}\right\|_{H^{1}}^{2}\right)$ for a general core-shell structure with piecewise constant coefficients. Without the core, it is shown in [14] that there is no connection between the blow up of the power and the localized resonance in general. In the works mentioned, the localized resonance was considered only in the cases where the settings are simple enough so that the separation of variables can be applied.

The paper is organized as follows. In Section 2, we state and prove several useful lemmas which are used in the proof of Theorem 1. The proof of Theorem 1 is presented in Section 3.

\section{Preliminaries}

In this section, we present some lemmas which will be used in the proof of Theorem 1 . The first one is on a three spheres inequality for Lipschitz matrix-valued functions.

Lemma 1 (Three spheres inequality). Let $d=2,3,0<R_{1}<R_{2}<R_{3}$ and let $M$ be a Lipschitz matrix-valued function defined in $B_{R_{3}}$ such that $M$ is uniformly elliptic in $B_{R_{3}}, M(0)=I$, and $M(x)=I$ for $x \in B_{R_{3}} \backslash B_{R_{2} / 2}$. Let $v \in$ $H^{1}\left(B_{R_{3}}\right)$ be a solution to the equation

$$
\operatorname{div}(M \nabla v)=0 \text { in } B_{R_{3}} .
$$

Then for all $0<\alpha<1$, there exists $R_{*}$ depending only on $R_{1}, R_{2}$, and the Lipschitz and ellipticity constants of $M$ such that, for $R_{3}>R_{*}$,

$$
\|v\|_{L^{2}\left(B_{R_{2}}\right)} \leq C\|v\|_{L^{2}\left(B_{R_{1}}\right)}^{\alpha}\|v\|_{L^{2}\left(B_{R_{3}}\right)}^{1-\alpha},
$$

for some positive constant $C$ independent of $v$.

Proof. By [1, Theorem 2.3 and (2.10)], there exists a constant $0<\beta<1$ which depends only on $R_{1}$ and $R_{2}$ such that

$$
\|v\|_{L^{2}\left(B_{R_{2}}\right)} \leq C\|v\|_{L^{2}\left(B_{R_{1}}\right)}^{\beta}\|v\|_{L^{2}\left(B_{2 R_{2}}\right)}^{1-\beta} .
$$

Here and in what follows in this proof, $C$ denotes a positive constant which is independent of $v$ and can change from one place to another. We claim that

$$
N\left(v, 2 R_{2}\right) \leq C N\left(v, R_{2} / 2\right)^{\gamma} N\left(v, R_{3} / 2\right)^{1-\gamma},
$$

where

$$
N(v, r):=\|v\|_{H^{1 / 2}\left(\partial B_{r}\right)}+\left\|\partial_{r} v\right\|_{H^{-1 / 2}\left(\partial B_{r}\right)}
$$

\footnotetext{
2 The terminology "oscillation" has been frequently used in the physics literature to mention the localized resonance. Here we use the standard terminology from mathematics community: "singularity". Both of them would be fine if one looks at the removed term $\hat{u}_{\delta}$ defined in (3.31) in the proof of Theorem 1 .
} 
and

$$
\gamma=\ln \left[R_{3} /\left(4 R_{2}\right)\right] / \ln \left[R_{3} / R_{2}\right] .
$$

The proof of (2.3) can be proceeded as follows. We only consider the case $d=2$, the case $d=3$ follows similarly. Since $\Delta v=0$ in $B_{R_{3}} \backslash B_{R_{2} / 2}$, one can represent $v$ in $B_{R_{3}} \backslash B_{R_{2} / 2}$ as follows

$$
v(x)=\sum_{n \geq 0} \sum_{ \pm}\left(a_{n, \pm} r^{n, \pm}+b_{n} r^{-n}\right) e^{ \pm i n \theta}
$$

for $a_{n, \pm}, b_{n, \pm} \in \mathbb{C}(n \geq 0)$ with the convention $a_{0,+}=a_{0,-}=b_{0,+}=b_{0,-}$. For $R_{2} / 2 \leq r \leq R_{3}$, we have

$$
N(v, r)^{2} \sim \sum_{n \geq 0} \sum_{ \pm}(n+1)\left(\left|a_{n, \pm}\right|^{2} r^{2 n}+\left|b_{n, \pm}\right|^{2} r^{-2 n}\right) .
$$

Here for two nonnegative quantities $\tau_{1}$ and $\tau_{2}, \tau_{1} \sim \tau_{2}$ means that $\tau_{1} \leq C \tau_{2}$ and $\tau_{2} \leq C \tau_{1}$. It follows that, for $R_{2} / 2 \leq$ $r_{1}<r_{2}<r_{3} \leq R_{3}$,

$$
N\left(v, r_{2}\right) \leq C N\left(v, r_{1}\right)^{\lambda} N\left(v, r_{3}\right)^{1-\lambda},
$$

where $\lambda=\lambda\left(r_{1}, r_{2}, r_{3}\right):=\ln \left(r_{3} / r_{1}\right) / \ln \left(r_{3} / r_{2}\right)$. We obtain claim (2.3).

A combination of (2.2) and (2.3) yields

$$
\|v\|_{L^{2}\left(B_{R_{2}}\right)} \leq C\|v\|_{L^{2}\left(B_{R_{1}}\right)}^{\frac{\beta}{1-\gamma(-\beta)}}\|v\|_{L^{2}\left(B_{R_{3}}\right)}^{\frac{(1-\beta)(1-\gamma)}{1-\gamma(1-\beta)}} .
$$

Here we use the fact that

$$
\|v\|_{L^{2}\left(B_{2 R_{2}}\right)} \leq C N\left(v, 2 R_{2}\right), \quad N\left(v, R_{2} / 2\right) \leq C\|v\|_{L^{2}\left(B_{R_{2}}\right)}, \quad \text { and } \quad N\left(v, R_{3} / 2\right) \leq C\|v\|_{L^{2}\left(B_{R_{3}}\right)} .
$$

By taking $R_{3}$ large enough so that $\gamma$ is close to $1, \frac{(1-\beta)(1-\gamma)}{1-\gamma(1-\beta)}$ is close to 0 . The conclusion follows.

The second lemma of this section is standard and its proof is left to the reader.

Lemma 2. Let $d \geq 2, \alpha, \beta>0, D \subset \subset \Omega$ be two smooth open subsets of $\mathbb{R}^{d}$, and $f \in L^{2}(\Omega), g \in H^{1 / 2}(\partial \Omega)$, and $h \in H^{-1 / 2}(\partial D)$. Assume that $v \in H^{1}(\Omega \backslash \partial D)$ is such that

$$
\Delta v=f \text { in } \Omega \backslash \partial D, \quad v=0 \text { on } \partial \Omega,
$$

and

$$
[v]=g \text { on } \partial D, \quad \text { and } \quad\left[\partial_{\eta} v\right]=h \text { on } \partial D .
$$

We have

$$
\|v\|_{H^{1}(\Omega \backslash \bar{D})}+\|v\|_{H^{1}(D)} \leq C\left(\|f\|_{L^{2}(\Omega)}+\|g\|_{H^{1 / 2}(\partial D)}+\|h\|_{H^{-1 / 2}(\partial D)}\right),
$$

for some positive constant $C$ independent of $f, g$, and $h$.

Here and in what follows, $\left.v\right|_{+},\left.v\right|_{-}$, and $[v]$ on $\partial D$ denote the trace of a function $v$ from the exterior, interior of $D$, and $\left.v\right|_{+}-\left.v\right|_{-}$on $\partial D$ respectively. Similarly, the notations $\left.\mathcal{M} \nabla v \cdot \eta\right|_{+},\left.\mathcal{M} \nabla v \cdot \eta\right|_{-}$, and $[\mathcal{M} \nabla v \cdot \eta]$ are used on $\partial D$ for an appropriate matrix $\mathcal{M}$ and an appropriate function $v$, where $\eta$ is the normal unit vector directed to the exterior of $D$.

The following lemma which is a consequence of [11, Lemma 4] is used in the proof of Theorem 1.

Lemma 3. Let $d \geq 2,0<R_{1}<R_{2}<R_{3}$ with $R_{3}=R_{2}^{2} / R_{1}, a \in\left[L^{\infty}\left(B_{R_{3} \backslash R_{2}}\right)\right]^{d \times d}$ be a matrix valued function, and $K: B_{R_{2}} \backslash \bar{B}_{R_{1}} \rightarrow B_{R_{3}} \backslash \bar{B}_{R_{2}}$ be the Kelvin transform with respect to $\partial B_{R_{2}}$, i.e.,

$$
K(x)=R_{2}^{2} x /|x|^{2} .
$$


For $v \in H^{1}\left(B_{R_{2}} \backslash B_{R_{1}}\right)$, define $w=v \circ F^{-1}$. Then

$$
\operatorname{div}(a \nabla v)=0 \text { in } B_{R_{2}} \backslash B_{R_{1}}
$$

if and only if

$$
\operatorname{div}\left(K_{*} a \nabla w\right)=0 \text { in } B_{R_{3}} \backslash B_{R_{2}} .
$$

Moreover,

$$
w=v \quad \text { and } \quad K_{*} a \nabla w \cdot \eta=-a \nabla v \cdot \eta \text { on } \partial B_{R_{2}} .
$$

\section{Proof of Theorem 1}

Let $\mathcal{A}$ be a matrix valued function defined in $B_{r_{3}}$ such that $\mathcal{A}=A=b$ in $B_{r_{3}} \backslash B_{r_{2}}, \mathcal{A}(0)=I$, and $\mathcal{A}$ is Lipschitz. Take $\ell>4$ large enough such that (2.1) holds for

$$
\alpha=2 / 3,
$$

with $M=\mathcal{A}, R_{1}=r_{2}, R_{2}=4 r_{2}$, and $R_{3}=r_{3}$. Then

$$
\|v\|_{L^{2}\left(B_{4 r_{2}}\right)} \leq\|v\|_{L^{2}\left(B_{r_{2}}\right)}^{\alpha}\|v\|_{L^{2}\left(B_{r_{3}}\right)}^{1-\alpha},
$$

for $v \in H^{1}\left(B_{r_{3}}\right)$ which satisfies $\operatorname{div}(\mathcal{A} \nabla v)=0$ in $B_{r_{3}}$.

Multiplying (1.7) by $\bar{u}_{\delta}$ and integrating on $\Omega$, we obtain

$$
\int_{\Omega}\left\langle s_{\delta} A \nabla u_{\delta}, \nabla u_{\delta}\right\rangle=-\int_{\Omega} f \bar{u}_{\delta} .
$$

Considering the imaginary part and the real part, we have

$$
\int_{\Omega}\left|\nabla u_{\delta}\right|^{2} \leq C \delta^{-1}\|f\|_{L^{2}(\Omega)}\left\|u_{\delta}\right\|_{L^{2}\left(\Omega \backslash B_{r_{3}}\right)} .
$$

In this proof, $C$ denotes a positive constant changing from one place to another but independent of $\delta$ and $f$. Since $u_{\delta}=0$ on $\partial \Omega$, it follows that

$$
\left\|u_{\delta}\right\|_{L^{2}(\Omega)} \leq C\left\|\nabla u_{\delta}\right\|_{L^{2}(\Omega)} .
$$

We derive from (3.3) that

$$
\left\|u_{\delta}\right\|_{H^{1}(\Omega)} \leq C \delta^{-1 / 2}\|f\|_{L^{2}(\Omega)}^{1 / 2}\left\|u_{\delta}\right\|_{L^{2}\left(\Omega \backslash B_{r_{3}}\right)}^{1 / 2}
$$

and

$$
\left\|u_{\delta}\right\|_{H^{1}(\Omega)} \leq C \delta^{-1}\|f\|_{L^{2}(\Omega)} .
$$

As in [11], let $u_{1, \delta}$ be the reflection of $u_{\delta}$ through $\partial B_{r_{2}}$ by $F$, i.e.,

$$
u_{1, \delta}=u_{\delta} \circ F^{-1} \text { in } \mathbb{R}^{d} \backslash \bar{B}_{r_{2}} .
$$

Applying Lemma 3 and using the fact that $F_{*} A=A$ in $B_{r_{3}} \backslash B_{r_{2}}$, we obtain

$$
u_{1, \delta}=\left.u_{\delta}\right|_{+} \text {on } \partial B_{r_{2}} \quad \text { and } \quad(1-i \delta) A \nabla u_{1, \delta} \cdot \eta=\left.A \nabla u_{\delta} \cdot \eta\right|_{+} \text {on } \partial B_{r_{2}} .
$$

Define $U_{\delta}$ in $B_{r_{3}}$ as follows

$$
U_{\delta}=\left\{\begin{array}{cl}
u_{\delta}-u_{1, \delta} & \text { in } B_{r_{3}} \backslash B_{r_{2}}, \\
0 & \text { in } B_{r_{2}} .
\end{array}\right.
$$


Using the fact that $F_{*} A=A$ in $B_{r_{3}} \backslash B_{r_{2}}$ and applying Lemma 3, we derive from (3.6), (3.7), and (3.8) that $U_{\delta} \in$ $H^{1}\left(B_{r_{3}}\right)$,

$$
\operatorname{div}\left(\mathcal{A} \nabla U_{\delta}\right)=0 \text { in } B_{r_{3}} \backslash \partial B_{r_{2}}, \quad \text { and } \quad\left[\mathcal{A} \nabla U_{\delta} \cdot \eta\right]=-\left.\frac{i \delta}{1-i \delta} \mathcal{A} \nabla u_{\delta} \cdot \eta\right|_{+} \text {on } \partial B_{r_{2}} .
$$

It is clear from (3.4), (3.6), and (3.8) that

$$
\left\|U_{\delta}\right\|_{H^{1}\left(B_{r_{3}}\right)} \leq C \delta^{-1 / 2}\|f\|_{L^{2}(\Omega)}^{1 / 2}\left\|u_{\delta}\right\|_{L^{2}\left(\Omega \backslash B_{r_{3}}\right)}^{1 / 2} .
$$

Let $w_{\delta} \in H_{0}^{1}\left(B_{r_{3}}\right)$ be the unique solution to

$$
\operatorname{div}\left(\mathcal{A} \nabla w_{\delta}\right)=0 \text { in } B_{r_{3}} \backslash \partial B_{r_{2}} \quad \text { and } \quad\left[\mathcal{A} \nabla w_{\delta} \cdot \eta\right]=-\left.\frac{i \delta}{1-i \delta} \mathcal{A} \nabla u_{\delta} \cdot \eta\right|_{+} \text {on } \partial B_{r_{2}} .
$$

Then

$$
\left\|w_{\delta}\right\|_{H^{1}\left(B_{r_{3}}\right)} \leq C \delta\left\|\left.A \nabla u_{\delta} \cdot \eta\right|_{+}\right\|_{H^{-1 / 2}\left(\partial B_{r_{2}}\right)} .
$$

Since

$$
\left\|\left.A \nabla u_{\delta} \cdot \eta\right|_{+}\right\|_{H^{-1 / 2}\left(\partial B_{r_{2}}\right)} \leq C\left\|u_{\delta}\right\|_{H^{1}(\Omega)},
$$

it follows from (3.4) and (3.12) that

$$
\left\|w_{\delta}\right\|_{H^{1}\left(B_{r_{3}}\right)} \leq C \delta^{1 / 2}\|f\|_{L^{2}(\Omega)}^{1 / 2}\left\|u_{\delta}\right\|_{L^{2}\left(\Omega \backslash B_{r_{3}}\right)}^{1 / 2} .
$$

Define

$$
V_{\delta}=U_{\delta}-w_{\delta} \text { in } B_{r_{3}} .
$$

Then $V_{\delta} \in H^{1}\left(B_{r_{3}}\right)$ is a solution to

$$
\operatorname{div}\left(\mathcal{A} \nabla V_{\delta}\right)=0 \text { in } B_{r_{3}} .
$$

Using (3.2), we obtain

$$
\left\|V_{\delta}\right\|_{L^{2}\left(B_{4 r_{2}}\right)} \leq C\left\|V_{\delta}\right\|_{L^{2}\left(B_{r_{2}}\right)}^{\alpha}\left\|V_{\delta}\right\|_{L^{2}\left(B_{r_{3}}\right)}^{1-\alpha} .
$$

From (3.8) and (3.14), we have

$$
V_{\delta}=-w_{\delta} \text { in } B_{r_{2}} .
$$

We derive from (3.13) that

$$
\left\|V_{\delta}\right\|_{L^{2}\left(B_{r_{2}}\right)} \leq C \delta^{1 / 2}\|f\|_{L^{2}(\Omega)}^{1 / 2}\left\|u_{\delta}\right\|_{L^{2}\left(\Omega \backslash B_{r_{3}}\right)}^{1 / 2} .
$$

On the other hand, from (3.10), (3.13), and (3.14), we have

$$
\left\|V_{\delta}\right\|_{L^{2}\left(B_{r_{3}}\right)} \leq C \delta^{-1 / 2}\|f\|_{L^{2}(\Omega)}^{1 / 2}\left\|u_{\delta}\right\|_{L^{2}\left(\Omega \backslash B_{r_{3}}\right)}^{1 / 2} .
$$

A combination of (3.16), (3.17), and (3.18) yields

$$
\left\|V_{\delta}\right\|_{L^{2}\left(B_{4 r_{2}}\right)}^{2} \leq C \delta^{(2 \alpha-1)}\|f\|_{L^{2}(\Omega)}\left\|u_{\delta}\right\|_{L^{2}\left(\Omega \backslash B_{r_{3}}\right)} .
$$

Since $\Delta V_{\delta}=0$ in $B_{4 r_{2}} \backslash B_{2 r_{2}}$, it follows that

$$
\left\|V_{\delta}\right\|_{H^{1 / 2}\left(\partial B_{3 r_{2}}\right)}^{2}+\left\|\partial_{r} V_{\delta}\right\|_{H^{-1 / 2}\left(\partial B_{3 r_{2}}\right)}^{2} \leq C \delta^{(2 \alpha-1)}\|f\|_{L^{2}(\Omega)}\left\|u_{\delta}\right\|_{L^{2}\left(\Omega \backslash B_{r_{3}}\right)} .
$$

A combination of (3.8), (3.13), (3.14), and (3.19) implies

$$
\left\|u_{\delta}-u_{1, \delta}\right\|_{H^{1 / 2}\left(\partial B_{3 r_{2}}\right)}^{2}+\left\|\partial_{r} u_{\delta}-\partial_{r} u_{1, \delta}\right\|_{H^{-1 / 2}\left(\partial B_{3 r_{2}}\right)}^{2} \leq C \delta^{2 \beta}\|f\|_{L^{2}(\Omega)}\left\|u_{\delta}\right\|_{L^{2}\left(\Omega \backslash B_{r_{3}}\right)},
$$


where, by (3.1),

$$
\beta:=(2 \alpha-1) / 2=1 / 6>0 .
$$

As in [11], let $u_{2, \delta}$ be the reflection of $u_{1, \delta}$ through $\partial B_{r_{3}}$ by $G$, i.e.,

$$
u_{2, \delta}=u_{1, \delta} \circ G^{-1} \text { in } B_{r_{3}} .
$$

We have

$$
\Delta u_{1, \delta}=0 \text { in } B_{r_{3}} \backslash B_{3 r_{2}} \text { and } \quad \Delta u_{2, \delta}=0 \text { in } B_{r_{3}},
$$

by $(1.11)$.

We next consider the case $d=2$ and the case $d=3$ separately.

Case 1: $d=2$. From (3.22), one can represent $u_{1, \delta}$ and $u_{2, \delta}$ as follows

$$
u_{1, \delta}=c_{0}+d_{0} \ln r+\sum_{n=1}^{\infty} \sum_{ \pm}\left(c_{n, \pm} r^{n}+d_{n, \pm} r^{-n}\right) e^{ \pm i n \theta} \text { in } B_{r_{3}} \backslash B_{3 r_{2}},
$$

and

$$
u_{2, \delta}=e_{0}+\sum_{n=1}^{\infty} \sum_{ \pm} e_{n, \pm} r^{n} e^{ \pm i n \theta} \text { in } B_{r_{3}},
$$

for $c_{0}, d_{0}, e_{0}, c_{n, \pm}, d_{n, \pm}, e_{n, \pm} \in \mathbb{C}(n \geq 1)$. We derive from (3.4) that

$$
\left|e_{0}\right|^{2}+\sum_{n=1}^{\infty} \sum_{ \pm} n\left|e_{n, \pm}\right|^{2} r_{3}^{2 n} \leq C \delta^{-1}\|f\|_{L^{2}(\Omega)}\left\|u_{\delta}\right\|_{L^{2}\left(\Omega \backslash B_{r_{3}}\right)} .
$$

Using the fact that $G_{*} F_{*} A=I$ and applying Lemma 3, we have

$$
u_{1, \delta}=u_{2, \delta} \text { on } \partial B_{r_{3}} \quad \text { and }\left.\quad(1-i \delta) \partial_{\eta} u_{1, \delta}\right|_{-}=\partial_{\eta} u_{2, \delta} \text { on } \partial B_{r_{3}} .
$$

A combination of (3.23), (3.24), and (3.26) yields

$$
c_{n, \pm} r_{3}^{n}+d_{n, \pm} r_{3}^{-n}=e_{n, \pm} r_{3}^{n}, \quad c_{n, \pm} r_{3}^{n}-d_{n, \pm} r_{3}^{-n}=\frac{1}{1-i \delta} e_{n, \pm} r_{3}^{n} \text { for } n \geq 1,
$$

and

$$
c_{0}=e_{0}, \quad d_{0}=0 .
$$

We derive from (3.27) that

$$
c_{n, \pm}=\frac{2-i \delta}{2(1-i \delta)} e_{n, \pm} \quad \text { and } \quad d_{n, \pm}=\frac{-i \delta}{2(1-i \delta)} r_{3}^{2 n} e_{n, \pm} \quad \text { for } n \geq 1 .
$$

From (3.28) and (3.29), we obtain

$$
u_{1, \delta}-u_{2, \delta}=\sum_{n=1}^{\infty} \sum_{ \pm} \frac{i \delta}{2(1-i \delta)}\left(r^{n}-\frac{r_{3}^{2 n}}{r^{n}}\right) e_{n, \pm} e^{ \pm i n \theta} .
$$

We now introduce the technique of removing localized singularity. Set

$$
\hat{u}_{\delta}:=\sum_{n=1}^{\infty} \sum_{ \pm} \frac{-i \delta}{2(1-i \delta)} e_{n, \pm} \frac{r_{3}^{2 n}}{r^{n}} e^{ \pm i n \theta} \text { in } \mathbb{R}^{2} \backslash B_{3 r_{2}} .
$$

Define $^{3}$

\footnotetext{
3 We remove $\hat{u}_{\delta}$ from $u_{\delta}$ in $B_{r_{3}} \backslash B_{3 r_{2}}$. Function $\hat{u}_{\delta}$ contains high modes and creates a trouble for estimating $u_{\delta}-u_{2, \delta}$ on $\partial B_{3 r_{2}}$ (to obtain an estimate for $u_{\delta}$ ). However this term can be negligible for large $|x|=r_{3}$ since $r^{-n}$ is small for large $r$. This is the reason for the choice of $\hat{u}_{\delta}$ and this also explains the terminology: "removing localized singularity".
} 


$$
W_{\delta}=\left\{\begin{array}{cl}
u_{\delta} & \text { in } \Omega \backslash B_{r_{3}} \\
u_{\delta}-\hat{u}_{\delta} & \text { in } B_{r_{3}} \backslash B_{3 r_{2}}, \\
u_{2, \delta} & \text { in } B_{3 r_{2}} .
\end{array}\right.
$$

It is clear from (3.22) that

$$
\Delta W_{\delta}=f \text { in } \Omega \backslash\left(\partial B_{r_{3}} \cup \partial B_{3 r_{2}}\right) .
$$

We claim that

$$
\left\|\hat{u}_{\delta}\right\|_{H^{1 / 2}\left(\partial B_{r_{3}}\right)}+\left\|\partial_{r} \hat{u}_{\delta}\right\|_{H^{-1 / 2}\left(\partial B_{r_{3}}\right)}=o(1)\|f\|_{L^{2}(\Omega)}+o(1)\left\|u_{\delta}\right\|_{L^{2}\left(\Omega \backslash B_{r_{3}}\right)} .
$$

Here and in what follows we use the standard notation: $o(1)$ denotes a quantity which converges to 0 as $\delta \rightarrow 0$. Indeed, from (3.25), we have

$$
\sum_{n=1}^{\infty} \sum_{ \pm} n \delta^{2}\left|e_{n, \pm}\right|^{2} r_{3}^{2 n} \leq C \delta\|f\|_{L^{2}(\Omega)}\left\|u_{\delta}\right\|_{L^{2}\left(\Omega \backslash B_{r_{3}}\right)} \leq o(1)\|f\|_{L^{2}}^{2}+o(1)\left\|u_{\delta}\right\|_{L^{2}\left(\Omega \backslash B_{r_{3}}\right.}^{2} .
$$

Hence (3.33) follows. We derive from (3.33) that

$$
\left\|\left[W_{\delta}\right]\right\|_{H^{1 / 2}\left(\partial B_{r_{3}}\right)}+\left\|\left[\partial_{r} W_{\delta}\right]\right\|_{H^{-1 / 2}\left(\partial B_{r_{3}}\right)}=o(1)\|f\|_{L^{2}(\Omega)}+o(1)\left\|u_{\delta}\right\|_{L^{2}\left(\Omega \backslash B_{r_{3}}\right)} .
$$

We next consider the transmission conditions for $W_{\delta}$ on $\partial B_{3 r_{2}}$. Since

$$
\left[W_{\delta}\right]=u_{\delta}-\hat{u}_{\delta}-u_{2, \delta} \quad \text { on } \partial B_{3 r_{2}},
$$

it follows from (3.30) and (3.31) that

$$
\left[W_{\delta}\right]=\left(u_{\delta}-u_{1, \delta}\right)+\sum_{n=1}^{\infty} \sum_{ \pm} \frac{i \delta}{2(1-i \delta)}\left(3 r_{2}\right)^{n} e_{n, \pm} e^{i n \theta} \quad \text { on } \partial B r_{2} .
$$

This implies

$$
\begin{aligned}
C\left\|\left[W_{\delta}\right]\right\|_{H^{1 / 2}\left(\partial B_{3 r_{2}}\right)}^{2} \leq & \left\|u_{\delta}-u_{1, \delta}\right\|_{H^{1 / 2}\left(\partial B_{3 r_{2}}\right)}^{2} \\
& +\sum_{n=1}^{\infty} \sum_{ \pm} \delta^{2} n\left|e_{n, \pm}\right|^{2} r_{3}^{2 n} \frac{\left(3 r_{2}\right)^{2 n}}{r_{3}^{2 n}}
\end{aligned}
$$

We derive from (3.20) and (3.25) that

$$
C\left\|\left[W_{\delta}\right]\right\|_{H^{1 / 2}\left(\partial B_{\left.3 r_{2}\right)}\right.}^{2} \leq \delta^{2 \beta}\|f\|_{L^{2}(\Omega)}\left\|u_{\delta}\right\|_{L^{2}\left(\Omega \backslash B_{R}\right)}+\delta\|f\|_{L^{2}(\Omega)}\left\|u_{\delta}\right\|_{L^{2}\left(\Omega \backslash B_{r_{3}}\right.} ;
$$

which yields

$$
\mid\left[W_{\delta}\right]\left\|_{H^{1 / 2}\left(\partial B_{\left.3 r_{2}\right)}\right)}^{2}=o(1)\right\| f\left\|_{L^{2}(\Omega)}^{2}+o(1)\right\| u_{\delta} \|_{L^{2}\left(\Omega \backslash B_{r_{3}}\right)}^{2} .
$$

Similarly,

$$
\left\|\left[\partial_{r} W_{\delta}\right]\right\|_{H^{-1 / 2}\left(\partial B_{3 r_{2}}\right)}^{2}=o(1)\|f\|_{L^{2}(\Omega)}^{2}+o(1)\left\|u_{\delta}\right\|_{L^{2}\left(\Omega \backslash B_{r_{3}}\right)}^{2} .
$$

Applying Lemma 2 and using (3.35), (3.37), and (3.38), we have

$$
\left\|W_{\delta}\right\|_{H^{1}\left(\Omega \backslash\left(\partial B_{r_{3}} \cup \partial B_{3 r_{2}}\right)\right)} \leq C\|f\|_{L^{2}},
$$

for small $\delta$.

Without loss of generality, one may assume that $W_{\delta} \rightarrow W$ weakly in $H^{1}\left(\Omega \backslash\left(\partial B_{r_{3}} \cup \partial B_{3 r_{2}}\right)\right)$ as $\delta \rightarrow 0$. From (3.35), (3.37), and (3.38), we have

$$
W \in H_{0}^{1}(\Omega) \quad \text { and } \quad \Delta W=f \text { in } \Omega .
$$


Hence $W=u$. Since the limit $W$ is unique, the convergence holds for the whole family $\left(W_{\delta}\right)$ as $\delta \rightarrow 0$. The proof is complete in two dimensions.

Case 2: $d=3$. The proof in the three dimensional case follows similarly as the one in the two dimensional case. We just note here that, in three dimensions, $u_{1, \delta}$ and $u_{2, \delta}$ can be represented, by (3.22), as follows

$$
u_{1, \delta}=c_{0}+\frac{d_{0}}{r}+\sum_{n=1}^{\infty} \sum_{k=-n}^{n}\left(c_{n, k} r^{n}+d_{n, k} r^{-n-1}\right) Y_{n}^{k}(x /|x|) \quad \text { in } B_{r_{3}} \backslash B_{r_{2}}
$$

and

$$
u_{2, \delta}=e_{0}+\sum_{n=1}^{\infty} \sum_{k=-n}^{n} e_{n, k} r^{n} Y_{n}^{k}(x /|x|) \quad \text { in } B_{r_{3}} .
$$

The proof is complete.

\section{Conflict of interest statement}

No conflict.

\section{Acknowledgements}

The author thanks Graeme Milton for pointing out the localized resonance from simulations in [8]. He also thanks Luca Rondi for enlightening discussions on the three spheres inequality.

\section{References}

[1] G. Alessandrini, L. Rondi, E. Rosset, S. Vessella, The stability for the Cauchy problem for elliptic equations, Inverse Probl. 25 (2009) 123004.

[2] A. Alu, N. Engheta, Achieving transparency with plasmonic and metamaterial coatings, Phys. Rev. E 95 (2005) 106623.

[3] H. Ammari, G. Ciraolo, H. Kang, H. Lee, G.W. Milton, Spectral theory of a Neumann-Poincaré-type operator and analysis of cloaking due to anomalous localized resonance, Arch. Ration. Mech. Anal. 218 (2013) 667-692.

[4] H. Ammari, G. Ciraolo, H. Kang, H. Lee, G.W. Milton, Anomalous localized resonance using a folded geometry in three dimensions, Proc. R. Soc. Lond. Ser. A 469 (2013) 20130048.

[5] H. Ammari, G. Ciraolo, H. Kang, H. Lee, G.W. Milton, Spectral theory of a Neumann-Poincaré-type operator and analysis of cloaking due to anomalous localized resonance II, Contemp. Math. 615 (2014) 1-14.

[6] G. Bouchitté, B. Schweizer, Cloaking of small objects by anomalous localized resonance, Q. J. Mech. Appl. Math. 63 (2010) $437-463$.

[7] R.V. Kohn, J. Lu, B. Schweizer, M.I. Weinstein, A variational perspective on cloaking by anomalous localized resonance, Commun. Math. Phys. (2014) 1-27.

[8] Y. Lai, H. Chen, Z. Zhang, C.T. Chan, Complementary media invisibility cloak that cloaks objects at a distance outside the cloaking shell, Phys. Rev. Lett. 102 (2009).

[9] G.W. Milton, N.P. Nicorovici, R.C. McPhedran, V.A. Podolskiy, A proof of superlensing in the quasistatic regime, and limitations of superlenses in this regime due to anomalous localized resonance, Proc. R. Soc. Lond. Ser. A 461 (2005) 3999-4034.

[10] G.W. Milton, N.-A.P. Nicorovici, On the cloaking effects associated with anomalous localized resonance, Proc. R. Soc. Lond. Ser. A 462 (2006) 3027-3059.

[11] H.M. Nguyen, Asymptotic behavior of solutions to the Helmholtz equations with sign changing coefficients, Trans. Am. Math. Soc. 367 (2015) 6581-6595.

[12] H.M. Nguyen, Superlensing using complementary media, Ann. Inst. Henri Poincaré, Anal. Non Linéaire 32 (2015) 471-484.

[13] H.M. Nguyen, Cloaking via anomalous localized resonance for doubly complementary media in the quasi static regime, J. Eur. Math. Soc. 17 (2015) 1327-1365.

[14] H.M. Nguyen, H.L. Nguyen, Complete resonance and localized resonance in plasmonic structures, ESAIM: Math. Model. Numer. Anal. 49 (2015) 741-754.

[15] N.A. Nicorovici, R.C. McPhedran, G.W. Milton, Optical and dielectric properties of partially resonant composites, Phys. Rev. B 49 (1994) $8479-8482$.

[16] J.B. Pendry, Negative refraction makes a perfect lens, Phys. Rev. Lett. 85 (2000) 3966-3969.

[17] R.A. Shelby, D.R. Smith, S. Schultz, Experimental verification of a negative index of refraction, Science 292 (2001) 77-79.

[18] V.G. Veselago, The electrodynamics of substances with simultaneously negative values of $\varepsilon$ and $\mu$, Usp. Fiz. Nauk 92 (1964) $517-526$. 